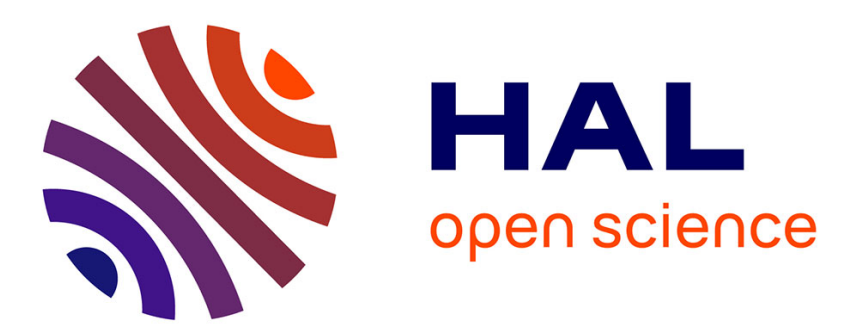

\title{
Selection of CMY-2 producing in the faecal flora of dogs treated with cephalexin
}

\author{
Peter Damborg, Ingrid B. Gaustad, John E. Olsen, Luca Guardabassi
}

\section{To cite this version:}

Peter Damborg, Ingrid B. Gaustad, John E. Olsen, Luca Guardabassi. Selection of CMY-2 producing in the faecal flora of dogs treated with cephalexin. Veterinary Microbiology, 2011, 151 (3-4), pp.404. 10.1016/j.vetmic.2011.03.015 . hal-00717089

\section{HAL Id: hal-00717089 \\ https://hal.science/hal-00717089}

Submitted on 12 Jul 2012

HAL is a multi-disciplinary open access archive for the deposit and dissemination of scientific research documents, whether they are published or not. The documents may come from teaching and research institutions in France or abroad, or from public or private research centers.
L'archive ouverte pluridisciplinaire HAL, est destinée au dépôt et à la diffusion de documents scientifiques de niveau recherche, publiés ou non, émanant des établissements d'enseignement et de recherche français ou étrangers, des laboratoires publics ou privés. 


\section{Accepted Manuscript}

Title: Selection of CMY-2 producing Escherichia coli in the faecal flora of dogs treated with cephalexin

Authors: Peter Damborg, Ingrid B. Gaustad, John E. Olsen, Luca Guardabassi

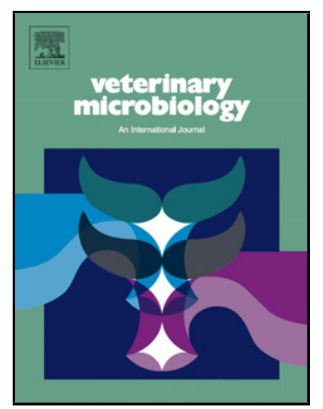

PII:

S0378-1135(11)00153-2

DOI: doi:10.1016/j.vetmic.2011.03.015

Reference: VETMIC 5238

To appear in:

VETMIC

Received date:

25-1-2011

Revised date:

$10-3-2011$

Accepted date:

$14-3-2011$

Please cite this article as: Damborg, P., Gaustad, I.B., Olsen, J.E., Guardabassi, L., Selection of CMY-2 producing Escherichia coli in the faecal flora of dogs treated with cephalexin, Veterinary Microbiology (2010), doi:10.1016/j.vetmic.2011.03.015

This is a PDF file of an unedited manuscript that has been accepted for publication. As a service to our customers we are providing this early version of the manuscript. The manuscript will undergo copyediting, typesetting, and review of the resulting proof before it is published in its final form. Please note that during the production process errors may be discovered which could affect the content, and all legal disclaimers that apply to the journal pertain. 

faecal flora of dogs treated with cephalexin

\author{
Peter Damborg*, Ingrid B. Gaustad, John E. Olsen and Luca Guardabassi
}

Department of Veterinary Disease Biology, Faculty of Life Sciences, University of Copenhagen, Stigbøjlen 4, 1870 Frederiksberg C., Denmark 


\section{Abstract}

29 Cephalexin is a first generation cephalosporin commonly used in dogs for treatment of pyoderma.

30 The objective of this study was to evaluate the in vivo effects of cephalexin on selection of

31 Escherichia coli resistant to extended-spectrum cephalosporins. A cohort study was conducted on

3213 dogs presenting clinical signs of pyoderma and treated with cephalexin and 22 healthy dogs that

33 had not been treated with antibiotics during the previous six months. Selective plating of faeces on

34 MacConkey agar plates containing cefotaxime (CTX) yielded growth of CTX-resistant E. coli for

35 eight of the 13 treated dogs $(62 \%)$, whereas no growth was observed for any of the control dogs

36 (Fisher Exact test, $P<0.001$ ). PCR and sequence analysis identified $b l a_{\mathrm{CMY}-2}$ in all eight dogs.

PCR-based replicon typing and restriction fragment length polymorphism (RFLP) of E. coli

transformants revealed location of $b l a_{\mathrm{CMY}-2}$ on indistinguishable IncI1 plasmids in five of the eight dogs. One representative of these five epidemiologically-related IncI1 plasmids was further characterized as sequence type (ST2) by plasmid multilocus sequence typing (pMLST). E. coli from the remaining three dogs harboured $b l a_{\mathrm{CMY}-2}$ on distinct plasmids with non-typeable replicons. A single isolate was classified as an extraintestinal pathogenic E. coli (ExPEC) due to the presence of $i u t A, p a p C$ and $s f a / f o c$. The results provide a strong indication that cephalexin selects for $E$. coli producing plasmid-borne CMY-2 $\beta$-lactamase. The isolation of a specific IncI1 plasmid carrying $b l a_{\mathrm{CMY}-2}$ from five epidemiologically unrelated dogs suggests that cephalexin use may contribute to the spread of this plasmid lineage among Danish dogs.

Keywords: antibiotic resistance, antibiotic therapy, AmpC, $\beta$-lactams, companion animals 


\section{Introduction}

A steady increase in usage of antimicrobial agents for companion animals, in particular for dogs, has been observed in Europe over the last years. In the UK, the total sales (kg of active ingredient) of antimicrobials registered for dogs increased by $250 \%$ in the period from 2004 to 2009 (Goodyear, 2010). Data from Denmark on total sales of antimicrobial agents for pet animals show a similar trend (Danmap, $2004 \&$ 2009). Much of this increase can be attributed to the frequent usage of cephalosporins and fluoroquinolones. In Sweden, prescription of these two antimicrobial classes in 1998-2005 has increased by $91 \%$ and 39\%, respectively (SVARM, 2005). Along with the increasing use of antibiotics, several important multi-resistant pathogens have emerged in dogs. Amongst Gram-negative species, various types of extended-spectrum $\beta$-lactamase genes have been described in Escherichia coli of canine origin, mainly bla $a_{\mathrm{CTX}-\mathrm{M}}$ (Carattoli et al., 2005b) and bla $a_{\mathrm{CMY}}$ (Sanchez et al., 2002).

In veterinary dermatology, cephalexin has been used for many years as the first choice in the management of pyoderma, one of the most frequent bacterial infections in the dog. According to a study in Finland (Rantala et al., 2004), cephalexin accounts for approximately $50 \%$ of all antibiotic prescriptions in dogs. On the contrary, the use of this first generation cephalosporin has been gradually abandoned in human medicine because of the limited antimicrobial activity against Gram-negative species. As a consequence, its impact on selection of extended-spectrum cephalosporin resistance in Gram-negative bacteria is largely unknown.

The present manuscript describes a cohort study designed to evaluate the possible selection of E. coli resistant to extended-spectrum cephalosporins in dogs treated with cephalexin. Treated and control dogs were screened for the occurrence of $E$. coli resistant to a third generation cephalosporin (cefotaxime), and the specific genes and plasmids mediating cephalosporin resistance were identified and characterized. 
 \\ 2. Materials and Methods}

\subsection{Study design and sampling}

Four small animal hospitals in the Greater Copenhagen area agreed to participate in the cohort study. Dogs attending these hospitals between February 2007 and March 2008 were considered eligible to the treatment group if they presented clinical signs of pyoderma, were prescribed minimum 14 days of standard oral treatment with cephalexin (Cefaseptin ${ }^{\circledR}$ Vet., $25 \mathrm{mg} / \mathrm{kg}$, BID) and did not receive any other medicine during treatment. Healthy dogs admitted to the same clinics for vaccination were enrolled in the control group if they had not received any antibiotics within 6 months prior to the study. Owners were asked to fill out a written consent of participation and send one fresh faecal sample per dog by ordinary mail to our laboratory upon hospital discharge. For the exposed dogs, samples were collected and sent on the last day of cephalexin treatment (day 14-28), whereas samples from control dogs were sent at the owners convenience in the week following vaccination.

\subsection{Bacterial isolation and identification}

Upon receipt in the laboratory, one gram of faeces was mixed with $700 \mu 150 \%$ glycerol and $300 \mu 1$ brain heart infusion (BHI) broth (Oxoid, Basingstoke, UK) and stored at $-80^{\circ} \mathrm{C}$ until processing. Samples were thawed and vortexed, and cefotaxime (CTX)-resistant coliforms were quantified by plate dilution. Briefly, $100 \mu \mathrm{l}$ from 10-fold dilutions made in Milli Q water was spread on MacConkey agar plates (Oxoid) supplemented with $2 \mu \mathrm{g} / \mathrm{ml}$ CTX. Following overnight incubation at $37^{\circ} \mathrm{C}$, lactose-positive colonies were counted, and one colony was randomly selected from each dog and subjected to the ImVIC phenotypic tests (indole, methyl red, Voges Proskauer and citrate) for $E$. coli identification. 


\subsection{Antimicrobial susceptibility}

Antimicrobial susceptibility testing of CTX-resistant $E$. coli was done by disk diffusion according to the Clinical Laboratories Standards Institute (CLSI, 2008a). The following discs (Oxoid) were used: amikacin (30 $\mu \mathrm{g})$, amoxicillin-clavulanic acid (20/10 $\mu \mathrm{g})$, ampicillin (10 $\mu \mathrm{g})$, aztreonam (30 $\mu \mathrm{g})$, cefepime $(30 \mu \mathrm{g})$, cefixime $(5 \mu \mathrm{g})$, cefotaxime $(30 \mu \mathrm{g})$, cefoxitin $(30 \mu \mathrm{g})$, ceftazidime $(30 \mu \mathrm{g})$, cephalothin (30 $\mu \mathrm{g})$, enrofloxacin $(5 \mu \mathrm{g})$, gentamicin $(10 \mu \mathrm{g})$, imipenem $(10 \mu \mathrm{g})$, sulfamethoxazoletrimethoprim 19:1 (25 $\mu \mathrm{g})$, and tetracycline (30 $\mu \mathrm{g})$. Susceptibility towards the combination of cefotaxime and clavulanic acid (30/10 $\mu \mathrm{g})$ was measured using BD BBL Sensi-Discs (Becton, Dickinson and Company, USA). Human CLSI breakpoints (CLSI, 2008b) were used for compounds without established breakpoints for bacteria of animal origin.

\subsection{Identification of $\beta$-lactamase genes}

PCR-based screening for the presence of bla $a_{\mathrm{CTX}-\mathrm{M}}$, bla $_{\mathrm{SHV}}$, bla $_{\mathrm{TEM}}$, and plasmid-borne genes encoding AmpC $\beta$-lactamase was done as previously described (Perez-Perez and Hanson, 2002; Hasman et al., 2005). Isolates positive for the AmpC phylogenetic group CIT were subjected to a specific PCR targeting the entire bla $a_{\mathrm{CMY}-2}$ gene (Pérez-Pérez and Hanson, 2002; Jørgensen et al., 2010). Amplicons were purified and submitted for sequencing to Macrogen Inc. (Seoul, Korea). The resulting nucleotide sequences were analysed using the basic local alignment search tool (BLAST) available on the homepage of the National Center for Biotechnology Information (http://www.ncbi.nlm.nih.gov/).

\subsection{Plasmid typing}

Plasmid DNA (Plasmid Midi Kit, Qiagen Inc., Venlo, The Netherlands) was obtained from all strains producing $\beta$-lactamase and transformed into electrocompetent Genehog E. coli (Invitrogen, 
Taastrup, Denmark). Transformants were selected on BHI agar plates supplemented with 2ug/ml CTX, and presence of $\beta$-lactamase genes was verified by colony PCR. Plasmid DNA from transformants was used as template in PCR-based replicon typing (Carattoli et al., 2005a) and for restriction fragment length polymorphism (RFLP) with the FastDigest ${ }^{\circledR}$ PstI enzyme (Fermentas, Helsingborg, Sweden). One IncI1 plasmid representing the unique RFLP pattern found was subjected to plasmid multilocus sequence typing (pMLST) as described by García-Fernández et al. (2008).

\subsection{Phylotyping and virulence gene profiling}

131 The phylotypes of E. coli isolates were determined by multiplex PCR (Clermont et al., 2001).

132 Another multiplex PCR was used to screen for the occurrence of virulence genes associated with 133 extraintestinal pathogenic E. coli (ExPEC) (Johnson et al., 2003). Isolates were defined as ExPEC if 134 they were PCR positive for at least two of the following genes: papA and/or papC, sfa/foc, afa/dra, kpsM II and iutA (Johnson et al., 2003)

\section{Results}

\subsection{Description of dogs in the treatment and control group}

139 A total of 13 dogs suffering from pyoderma and subjected to cephalexin treatment were included in

140 the treatment group. These dogs represented 10 breeds and were 1-11 years old (average 4.8 years).

141 Seven of the dogs were male and six were bitches. All dogs had been treated daily with cephalexin 142 for 14-28 days prior to sampling, and six of the dogs had been subjected to antimicrobial treatment 143 with either cephalexin $(n=4)$ or amoxicillin-clavulanate $(n=2)$ during the last year (Table 1$)$.

144 Twenty-two dogs were enrolled in the control group. They represented 15 breeds and were 1-7 145 years old (average 4.0 years). The control group included 11 males and 11 bitches. 


\subsection{Quantification and identification of CTX-resistant isolates}

148 CTX-resistant E. coli were isolated from eight dogs treated with cephalexin (62\%), whereas no 149 growth was obtained from dogs in the control group (Fisher exact test, $P<0.001$ ). Amongst the 150 positive dogs, counts of CTX-resistant coliforms ranged from $4 * 10^{7}$ to $4 * 10^{10} \mathrm{CFU} / \mathrm{g}$ faeces 151 (average $7 * 10^{9} \mathrm{CFU} / \mathrm{g}$ ). The eight selected isolates, one for each positive dog, displayed susceptibility profiles indicating presumptive production of AmpC-type $\beta$-lactamase (i.e. inactivity of clavulanic acid and resistance to all $\beta$-lactams except for cefepime and imipenem). All isolates were susceptible to amikacin, enrofloxacin and gentamicin. Three isolates displayed either intermediate or full resistance to sulfamethoxazole-trimethoprim, and one isolate was resistant to tetracycline (Table 1).

\subsection{Genotypic characterization of CTX-resistant E. coli}

All eight isolates carried $b l a_{\mathrm{CMY}-2}$. Replicon typing showed that this gene was present on an IncI1 plasmid in five of the eight isolates, whereas the remaining three isolates carried the gene on

161 plasmids that were non-typeable by replicon PCR. The IncI1 plasmids were indistinguishable by 162 RFLP analysis. One representative IncI1 plasmid from dog R7 (Table 1) was identified as sequence 163 type (ST) 2 by pMLST analysis. The extended-spectrum $\beta$-lactamase (ESBL)-encoding genes $164 b l a_{\mathrm{CTX}-\mathrm{M}}$ and $b l a_{\mathrm{SHV}}$ were not detected, whereas $b l a_{\mathrm{TEM}-1}$ was present in one isolate (Table 1). All 165 isolates belonged to phylotype $\mathrm{D}$, and one of the isolates was characterized as an ExPEC due to 166 simultaneous presence of the virulence genes iutA, papC and sfa/foc. 


\section{Discussion}

The present study shows that oral treatment of dogs with cephalexin results in high numbers of $E$. coli producing plasmid-mediated CMY-2 $\beta$-lactamase at the end of the treatment period. This information is noteworthy considering the extensive use of first-generation cephalosporins in small animal practice. CMY-2 confers resistance towards all $\beta$-lactams licensed for use in dogs and therefore may pose a serious animal health problem due to the limited alternative options available for treatment of $E$. coli infections in veterinary medicine. Although all the strains described in this study remained susceptible to most non- $\beta$-lactams tested (Table 1 ), multidrug-resistant E. coli producing CMY $\beta$-lactamase have been described as a cause of opportunistic infections in dogs (Sanchez et al., 2002; Sidjabat et al., 2006). Plasmids carrying $b l a_{\mathrm{CMY}-2}$ can easily acquire multiple resistance genes as suggested by frequent co-residence of different resistance genes (Winokur et al., 2001).

The results of this study suggest that dogs receiving prolonged treatment with firstgeneration cefalosporins may be an important reservoir of CMY $\beta$-lactamases. Sparse information available in the literature supports these findings. Murphy et al. (2009) isolated CMY-2-producing E. coli in 2 out of 180 healthy dogs attending veterinary hospitals in Canada. However, the prevalence of CMY-2 was likely underestimated as the study did not employ antimicrobial selective methods of isolation. In a recent study in the US (O'Keefe et al., 2010), CMY was detected in approximately one third of 150 canine and feline E. coli isolated from urinary tract infections and was by far the most common $\beta$-lactamase. Other studies have reported the occurrence of ESBL genes such as $b l a_{\mathrm{CTX}-\mathrm{M}-1}, b l a_{\mathrm{SHV}-12}$ or $b l a_{\mathrm{TEM}-52}$ in E. coli isolated from dogs in Portugal and Italy (Costa et al., 2004; Carattoli et al., 2005b). The apparent geographical variation in the distribution of $\beta$-lactamase types in dogs may be influenced by the methodologies used in different studies but 
could also reflect local patterns in the use of antimicrobials and in the epidemiology of $\beta$-lactamases in the human population.

Despite the sporadic occurrence of human infections caused by CMY-2-producing $E$. coli in Scandinavian countries (Jørgensen et al., 2010; Naseer et al., 2010), the presence of these bacteria in dogs may be of public health concern. One of the eight canine CMY-2-producing isolates described in this study (R6AC) was an ExPEC, which is the most common cause of urinary tract infections in humans (Johnson and Russo, 2001). Furthermore, all isolates belonged to phylotype D, which - together with phylotype B2 - is frequently shared between dogs and humans living in the same household (Damborg et al., 2009). In conjunction with our results, a strong association between CMY-2 and phylotype D has been recently demonstrated in E. coli infections in Spain (Oteo et al., 2010).

The $b l a_{\mathrm{CMY}-2}$ gene was located on IncI1 plasmids with identical RFLP patterns of which one representative belonged to ST2, in five of the eight $E$. coli isolates (Table 1). The predominance of one plasmid type was unexpected, since the dogs were epidemiologicallyunrelated and had been sampled on distinct dates at four different hospitals. This finding suggests that treatment with cephalexin may contribute to the spread of this plasmid lineage among dogs in Denmark. Unfortunately, no information is available on the type of plasmids carrying bla $_{\mathrm{CMY}-2}$ in human and canine E. coli infections in this country. IncI1 is the predominant plasmid incompatibility group among CMY-2-producing E. coli collected from humans in Norway (Naseer et al., 2010). According to the publically available database (http://pubmlst.org/plasmid), IncI1 ST2 plasmids carrying the bla $a_{\mathrm{CMY}-2}$ gene have been previously detected in $E$. coli from dogs in Italy and in E. coli and Salmonella Heidelberg from Canadian and UK citizens. Such a widespread appearance across hosts and continents indicates high mobility and broad host-range of this plasmid

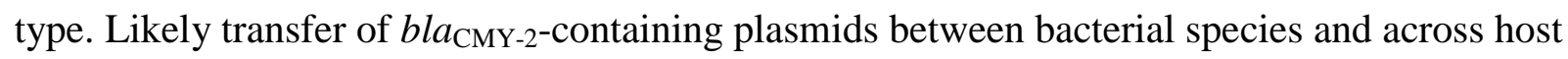


216 barriers has previously been hypothesized by Winokur et al. (2001). The gene has been also

217 reported in other plasmid incompatibility groups, primarily A/C and K/B (Carattoli, 2009; Mataseje

218 et al., 2010).

The observed association between cephalexin and CMY-2-producing E. coli could

have been enhanced by prior antimicrobial treatment, since at least six of the eight bla $_{\mathrm{CMY}-2 \text {-positive }}$ dogs had previously been treated with $\beta$-lactams, mainly cephalexin (Table 1 ). In contrast, only one of the five culture-negative dogs from the treatment group had received $\beta$-lactams before. Studies in humans have shown a long-term effect of antimicrobial treatment on resistance levels in the intestinal flora (Jernberg et al., 2007). In animals, studies have mainly focused on the short-term selective effects of antimicrobial treatment and their results are often controversial, maybe as a consequence of the different methodologies, animal species, antibiotics and resistant bacteria used for assessing the impact of antimicrobial treatment (Cavaco et al., 2008; Singer et al., 2008; Grønvold et al., 2010).

A limitation of the study was the initial storage of faecal samples in BHI and glycerol at $-80^{\circ} \mathrm{C}$. However, the high counts of CTX-resistant coliforms revealed that freezing did not affect bacterial survival, and even if there was an effect of storage, such an effect would have equally affected the bacterial counts in all samples. We can therefore conclude that this methodological factor did not influence the main outcome of the study. As we analysed a single CTX-resistant $E$. coli isolate per sample, $E$. coli producing other $\beta$-lactamases than CMY-2 may have gone undetected. However, isolation of multiple colonies from the same plate was unlikely to overcome this intrinsic methodological limitation of culture methods, since it has previously been shown that multiple $E$. coli isolates from the same faecal sample often represent one, dominant clone (McLellan et al., 2003). 
240 impacts the composition of the intestinal E. coli population in favour of resistant strains (Mentula et

241 al., 2004; Grønvold et al., 2010). However, such studies assessed the effects of $\beta$-lactam agents 242 other than cephalexin (ampicillin and amoxicillin, respectively) and did not investigate the genetic 243 background of the resistant strains selected by antimicrobial treatment. More in vivo studies are 244 needed to identify drug- and gene-specific associations between antimicrobial usage and 245 antimicrobial resistance. This information is of crucial importance to understand how the evolution 246 of antimicrobial resistance may be influenced by patterns of antimicrobial usage, and consequently 247 to develop effective antimicrobial policies in veterinary hospitals.

\section{Conclusion}

250 Oral treatment of dogs with cephalexin selects for intestinal E. coli producing CMY-2 and may 251 contribute to the dissemination of specific IncI1 plasmids encoding this $\beta$-lactamase. The intensive 252 use of cephalexin during the last decades may have favoured local dissemination of $b l a_{\mathrm{CMY}-2}$ in 253 Danish dogs.

\section{Acknowledgements}

256 We would like to thank staff at the veterinary hospitals Hospital for Mindre Husdyr (Frederiksberg), 257 Trollesminde Dyreklinik (Hillerød), Klinik for Veterinær Dermatologi (Lyngby) and Himmelev 258 Dyreklinik (Roskilde). We would also like thank dog owners for agreeing to participate in the 259 study. 


\section{References}

264 Carattoli, A., Bertini, A., Villa, L., Falbo, V., Hopkins, K.L., Threlfall, E.J., 2005a. Identification of 265 plasmids by PCR-based replicon typing. J. Microbiol. Meth. 63, 219-228.

267 Carattoli, A., Lovari, S., Franco, A., Cordaro, G., Di Matteo, P., Battisti, A., 2005b. Extended268 spectrum $\beta$-lactamases in Escherichia coli isolated from dogs and cats in Rome, Italy, from 2001 to 269 2003. Antimicrob. Agents Chemother. 49, 833-835.

271 Carattoli, A., 2009. Resistance plasmid families in Enterobacteriaceae. Antimicrob. Agents.

272 Chemother. 53, 2227-2238.

274 Cavaco, L.M., Abatih, E., Aarestrup, F.M., Guardabassi, L., 2008. Selection and persistence of 275 CTX-M-producing Escherichia coli in the intestinal flora of pigs treated with amoxicillin, ceftiofur, 276 or cefquinome. Antimicrob. Agents Chemother. 32, 3612-3616.

278 Clermont, O., Bonacorsi, S., Bingen, E., 2000. Rapid and simple determination of Escherichia coli 279 phylogenetic group, Appl. Environ. Microbiol. 66, 4555-4558.

281 Clinical Laboratory Standards Institute (CLSI), 2008a. Performance Standards for Antimicrobial 282 Disk and Dilution Susceptibility Tests for Bacteria Isolated from Animals; Approved standard 283 Third edition. M31-A3, vol. 28, no. 8, CLSI, Wayne, PA, USA. 
285 Clinical and Laboratory Standards Institute (CLSI), 2008b. Performance Standards for 286 Antimicrobial Susceptibility Testing: Eighteenth Informational Supplement M100-S18, vol. 28, no. 1. CLSI, Wayne, PA, USA.

Costa, D., Poeta, P., Brinas, L., Saenz, Y., Rodrigues, J., Torres, C., 2004. Detection of CTX-M-1 and TEM-52 beta-lactamases in Escherichia coli strains from healthy pets in Portugal. J. Antimicrob. Chemother. 54, 960-961.

Damborg, P., Nielsen, S.S., Guardabassi, L., 2009. Escherichia coli shedding patterns in humans and dogs: insight into within-household sharing of phylotypes associated with urinary tract infections. Epidemiol. Infect. 137, 1457-1464.

DANMAP, 2004 and 2009. Use of Antimicrobial Agents and Occurrence of Antimicrobial

Resistance in Bacteria from Food Animals, Foods and Humans in Denmark. Copenhagen,

Denmark, 2005 and 2010. http://www.vet.dtu.dk, ISSN 1600-2032.

301 García-Fernández, A., Chiaretto, G., Bertini, A., Villa, L., Fortini, D., Ricci, A., Carattoli, A., 2008. 302 Multilocus sequence typing of IncI1 plasmids carrying extended-spectrum beta-lactamases in 303 Escherichia coli and Salmonella of human and animal origin. J. Antimicrob. Chemother. 61, 12293041233.

306 Goodyear, K., 2010. Sales of antimicrobial products authorised for use as veterinary medicines, antiprotozoals, antifungals, growth promoters and coccidiostats in the UK in 2009. Veterinary 
308 Medicines Directorate. http://www.vmd.gov.uk/Publications/Antibiotic/salesanti09.pdf. Day last 309 visited: Nov $23^{\text {rd }}, 2010$.

311 Grønvold, A.R., L`Abée-Lund, T.M., Sørum, H., Skancke, E., Yannarell, A.C., Mackie, R.I., 2009. 312 Changes in fecal microbiota of healthy dogs administered amoxicillin. FEMS Microbiol. Ecol. 71, $313 \quad 313-326$.

315 Hasman H, Mevius D, Veldman K, Olesen I., Aarestrup, F.M., 2005. Beta-lactamases among 316 extended-spectrum beta-lactamase (ESBL)-resistant Salmonella from poultry, poultry products and 317 human patients in The Netherlands. J. Antimicrob. Chemother. 56, 115-121.

319 Jernberg, C., Löfmark, S., Edlund, C., Jansson, J.K., 2007. Long-term ecological impacts of 320 antibiotic administration on the human intestinal microbiota. ISME J. 1, 56-66.

Johnson, J.R., Gajewski, A., Lesse, A.J., Russo, T.A., 2003. Extraintestinal pathogenic Escherichia coli as a cause of invasive nonurinary infections. J. Clin. Microbiol. 41, 5798-5802.

Johnson, J.R., Russo, T.A. 2001. Extraintestinal pathogenic Escherichia coli: "the other bad E. coli”. J. Lab. Clin. Med. 139, 155-162.

Jørgensen R.L., Nielsen J.B., Friis-Moller A., Fjeldsoe-Nielsen, H., Schonning, K., 2010.

329 Prevalence and molecular characterization of clinical isolates of Escherichia coli expressing an 330 AmpC phenotype. J Antimicrob Chemother. 65, 460-464. 
Mataseje, L.F., Baudry, P.J., Zhanel, G.G., Morck, D.W., Read, R.R., Louie, M., Mulvey, M.R., 2010. Comparison of CMY-2 plasmids isolated from human, animal, and environmental Escherichia coli and Salmonella spp. from Canada. Diagn. Microbiol. Infect. Dis. 67, 387-391.

McLellan, S.L., Daniels, A.D., Salmore, A.K., 2003. Genetic characterization of Escherichia coli populations from host sources of fecal pollution by using DNA fingerprinting. Appl. Environ. Microbiol. 69, 2587-2594.

Mentula, S., Harmoinen, J., Koski, P., Westermarck, E., Rautio, M., Huovinen, P., Könönen, E., 2004. Inhibition of ampicillin-induced emergence of resistance in intestinal coliforms by targeted recombinant beta-lactamase. Int. J. Antimicrob. Agents 24, 555-561.

Murphy, C., Reid-Smith, R.J., Prescott, J.F., Bonnett, B.N., Poppe, C., Boerlin, P., Weese, J.S., Janecko, N., McEwen, S.A., 2009. Occurrence of antimicrobial resistant bacteria in healthy dogs and cats presented to private veterinary hospitals in southern Ontario: a preliminary study. Can. Vet. J. 50, 1047-1053.

Naseer, U., Haldorsen, B., Simonsen, G.S., Sundsfjord, A., 2010. Sporadic occurrence of CMY-2producing multidrug-resistant Escherichia coli of ST-complexes 38 and 448, and ST131 in Norway. Clin Microbiol. Infect. 16, 171-178.

O’Keefe, A., Hutton, T.A., Schifferli, D.M., Rankin, S.C., 2010. First detection of CTX-M and SHV extended-spectrum $\beta$-lactamases in Escherichia coli urinary tract isolates from dogs and cats in the United States. Antimicrob. Agents Chemother. 54, 3489-3492. 
356 Oteo, J., Cercenado, E., Cuevas, O., Bautista, V., Delgado-Iribarren, A., Orden, B., Pérez-Vázquez, 357 M., García-Cobos, S., Campos, J., 2010. AmpC beta-lactamases in Escherichia coli: emergence of 358 CMY-2-producing virulent phylogroup D isolates belonging mainly to STs $57,115,354,393$, and 359420 , and phylogroup B2 isolates belonging to the international clone O25b-ST131. Diagn.

360 Microbiol. Infect. Dis. 67, 270-276.

362 Pérez-Pérez F.J., Hanson, N.D., 2002. Detection of plasmid-mediated AmpC beta-lactamase genes 363 in clinical isolates by using multiplex PCR. J. Clin. Microbiol. 40, 2153-2162.

365 Rantala, M., Hölsö, K., Lillas, A., Huovinen, P., Kaartinen, L., 2004. Survey of condition-based 366 prescribing of antimicrobial drugs for dogs at a veterinary teaching hospital. Vet. Rec. 155, 259367262.

369 Sanchez, S., McCrackin Stevenson, M.A., Hudson, C.R., Maier, M., Buffington, T., Dam, Q., 370 Maurer, J.J., 2002. Characterization of multidrug-resistant Escherichia coli isolates associated with 371 nosocomial infections in dogs. J. Clin. Microbiol. 40, 3586-3595.

373 Sidjabat, H.E., Townsend, K.M., Hanson, N.D., Bell, J.M., Stokes, H.W., Gobius, K.S., Moss, 374 S.M., Trott, D.J., 2006. Identification of $b l a_{\mathrm{CMY}-7}$ and associated plasmid-mediated resistance genes 375 in multidrug-resistant Escherichia coli isolated from dogs at a veterinary teaching hospital in 376 Australia. J. Antimicrob. Chemother. 57, 840-848. 
378 Singer, R.S., Patterson, S.K., Wallace, R.L., 2008. Effects of therapeutic ceftiofur administration to

379 dairy cattle on Escherichia coli dynamics in the intestinal tract. Appl. Environ. Microbiol. 74, 69563806962.

382 SVARM, 2005. Swedish Veterinary Antimicrobial Resistance Monitoring. Uppsala, Sweden, 2006.

383 http://www.sva.se, ISSN 1650-6332.

384

385 Winokur, P.L., Vonstein, D.L., Hoffman, L.J., Uhlenhopp, E.K., Doern, G.V., 2001. Evidence for 386 transfer of CMY-2 AmpC $\beta$-lactamase plasmids between Escherichia coli and Salmonella isolates 387 from food animals and humans. Antimicrob. Agents Chemother. 45, 2716-2722. 
Table 1. Description of dogs and CTX-resistant E. coli isolates

\begin{tabular}{|c|c|c|c|c|c|c|c|c|}
\hline \multicolumn{3}{|c|}{ Dogs with CTX-resistant E. coli } & \multicolumn{6}{|c|}{ Characterization of CTX-resistant E. coli } \\
\hline Dog ID & $\begin{array}{c}\text { Days of } \\
\text { cephalexin } \\
\text { treatment }\end{array}$ & $\begin{array}{l}\text { Previous } \beta \text {-lactam treatment } \\
\text { (mth's before sampling) }\end{array}$ & $\begin{array}{l}\beta \text {-lactamase } \\
\text { gene }(s)\end{array}$ & $\begin{array}{l}\text { Plasmid } \\
\text { replicon type }\end{array}$ & $\begin{array}{c}\text { Plasmid } \\
\text { RFLP pattern }\end{array}$ & $\begin{array}{l}\text { Resistance to } \\
\text { non- } \beta \text {-lactams }^{\mathrm{b}}\end{array}$ & Phylotype & ExPEC \\
\hline R1 & 28 & $\begin{array}{c}\text { Cephalexin (8) } \\
\text { Amoxicillin-clavulanate (1) }\end{array}$ & $b l a_{\mathrm{CMY}-2}$ & Unknown & A & - & $\mathrm{D}$ & No \\
\hline $\mathrm{R} 2$ & 21 & Unknown & $b l a_{\mathrm{CMY}-2}$ & IncI1 & $\mathrm{B}$ & - & $\mathrm{D}$ & No \\
\hline R3 & 17 & Amoxicillin-clavulanate (9) & $b l a_{\mathrm{CMY}-2}$ & IncI1 & $\mathrm{B}$ & - & $\mathrm{D}$ & No \\
\hline $\mathrm{R} 4$ & 14 & Cephalexin (2) & $b l a_{\mathrm{CMY}-2}$ & Unknown & $\mathrm{C}$ & TET & $\mathrm{D}$ & No \\
\hline R5 & 14 & None & $b l a_{\mathrm{CMY}-2}$ & IncI1 & $\mathrm{B}$ & SXT & $\mathrm{D}$ & No \\
\hline R6 & 19 & $\begin{array}{l}\text { Cephalexin (9) } \\
\text { Cephalexin (3) }\end{array}$ & $b l a_{\mathrm{CMY}-2}$ & IncI1 & B & - & $\mathrm{D}$ & Yes \\
\hline R7 & 21 & Unknown & $b l a_{\mathrm{CMY}-2} b l a_{\mathrm{TEM}-1}$ & $\mathrm{IncI}^{\mathrm{a}}$ & $\mathrm{B}$ & SXT & $\mathrm{D}$ & No \\
\hline $\mathrm{R} 13$ & 15 & Unknown & $b l a_{\mathrm{CMY}-2}$ & Unknown & $\mathrm{D}$ & SXT & D & No \\
\hline
\end{tabular}

${ }^{\mathrm{a}}$ plasmid multilocus sequence type 2

${ }^{\mathrm{b}}$ TET, tetracycline; SXT, sulphamethoxazole-trimethoprim. 